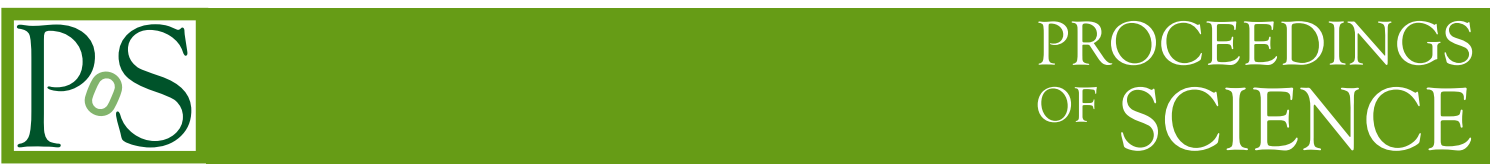

\title{
Artefacts due to bandpass mis-match
}

\section{J. Patrick Leahy}

University of Manchester

E-mail:

\section{Karen Foley}

National University of Ireland, Maynooth

E-mail: 'karen. foley@nuim.iè

Because of their large fractional bandwidth, CMB detectors do not map the sky at a well-defined frequency. If calibrated on the CMB dipole, the resulting maps are accurate for CMB emission, but not for foregrounds which have a different spectrum. Hence:

- Calibrated maps from different detectors at the same nominal frequency will not be consistent;

- Measurements of the foreground spectrum must allow for the bandwidth-induced errors in each detector (which depend on both the bandpass $g(v)$ and on the spectral index $\beta$ );

- Differences between the bandpasses for the two polarizations in a detector lead to noncancellation of the foreground total intensity in the ' $a$ ' - ' $b$ ' signal, and hence to a spurious polarization.

We show that, in some ways, the response of the radiometers to continuum emission is remarkably insensitive to the detailed bandpass structure, and can be modelled to high precision with an elementary 'top-hat' bandpass shape. However, it is not possible to accurately match the model parameters of different radiometers, leading to residual errors which can only be removed in tandem with modelling the foreground emission. We illustrate these effects by estimating their amplitude for the Planck LFI $30 \mathrm{GHz}$ channel.

$C M B$ and Physics of the Early Universe

20-22 April 2006

Ischia, Italy 


\section{Formalism}

Modern high-gain, high-bandwidth radiometers display substantial structure in their gain-

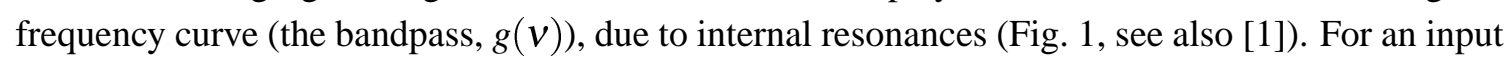
spectrum $T_{A}(v)$, the observed uncalibrated signal is $\int g(v) T_{A}(v) d v$. To ensure that the calibrated output is equal to the input antenna temperature at the nominal frequency $v_{0}$, when observing CMB fluctuations (writing $\Delta T_{A}=\eta_{\Delta T}(v) \Delta T$ ), we require

$$
T_{\mathrm{cal}}=\eta_{\Delta T}\left(v_{0}\right) \frac{\int g(v) T_{A}(v) d v}{\int g(v) \eta_{\Delta T}(v) d v}
$$

For any detector observing emission with $T_{A}=k v^{\beta}$, there is an effective frequency $v_{\text {eff }}(\beta)$ for which the temperature scale is correct, $T_{\text {cal }}\left(v_{0}=v_{\text {eff }}\right)=T_{A}\left(v_{\text {eff }}\right)$. Fig. 'in shows numerical solutions for $v_{\text {eff }}(\beta)$ for the example bandpasses of Fig. 11. But we cannot measure $\beta$ precisely, so even if we set $v_{0}=v_{\text {eff }}\left(\beta_{0}\right)$ for a typical foreground spectral index $\beta_{0}$, there is a residual gain error $f(\beta)$. Because the assumed foreground spectrum is very smooth, $f(\beta)$ can be accurately represented by a quadratic, as Fig. $\overline{\beta_{1}}$ shows, despite the complex structure of the bandpass.

\subsection{Analytic Model}

For a sharply cut off bandpass (limits $v_{1}$ and $v_{2}$ ), with arbitrary slope across the band, assuming a locally power-law spectrum for the CMB,

$$
f(\beta) \approx\left(\beta-\beta_{\mathrm{CMB}}\right)\left[\varepsilon+\frac{b^{2}}{24}\left(\beta-\beta_{0}\right)\right]
$$

where $\varepsilon$ is the fractional offset between $v_{0}$ and $v_{\text {eff }}\left(\beta_{0}\right)$, and $b=\ln \left(v_{2} / v_{1}\right)$ is the fractional bandwidth. Hence a frequency offset gives artefacts linearly proportional to the spectral index difference from the $\mathrm{CMB}$, while the finite bandwidth causes curvature in $f(\beta)$. For a real bandpass we can take $b$ and $\beta_{\mathrm{CMB}}$ as adjustable parameters: for our examples $b \sim 1.2(\Delta v / v)$ where $\Delta v$ is the usual
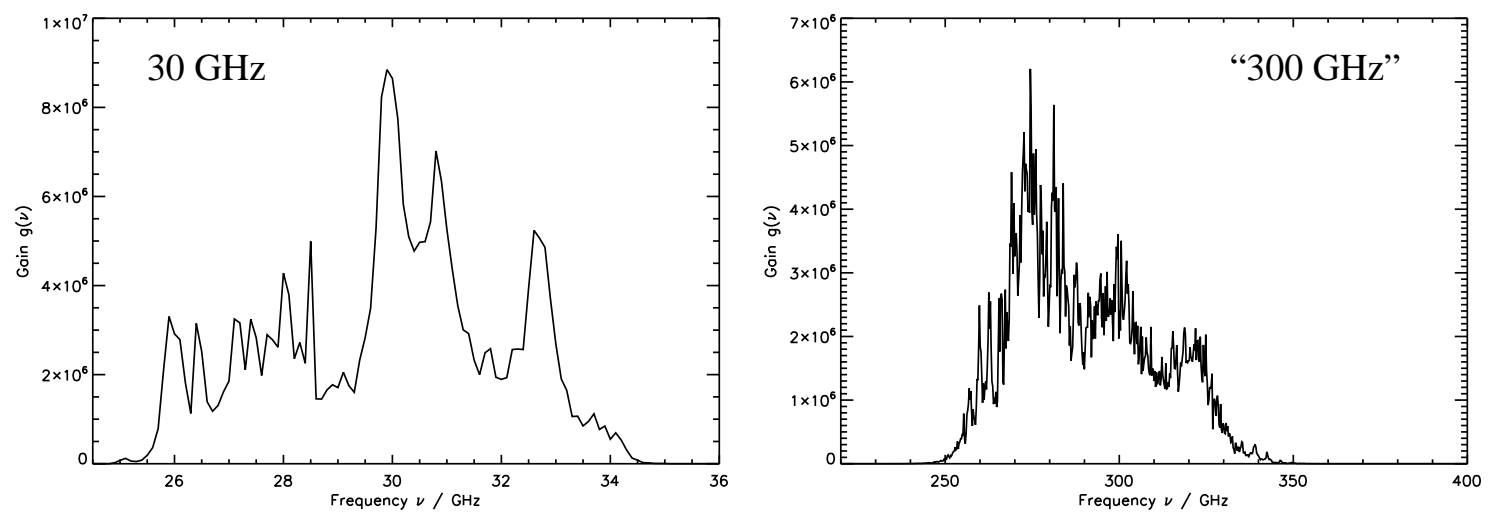

Figure 1: Examples of radiometer bandpasses $g(v)$ on a linear scale to emphasise the detailed structure (data courtesy D. Kettle). (a) Left: Prototype Planck $30 \mathrm{GHz}$ system. (b) Right, another bandpass artificially scaled to a nominal frequency of $300 \mathrm{GHz}$. The detailed structure varies from unit to unit, yielding different effective gains for foreground emission in each device. 

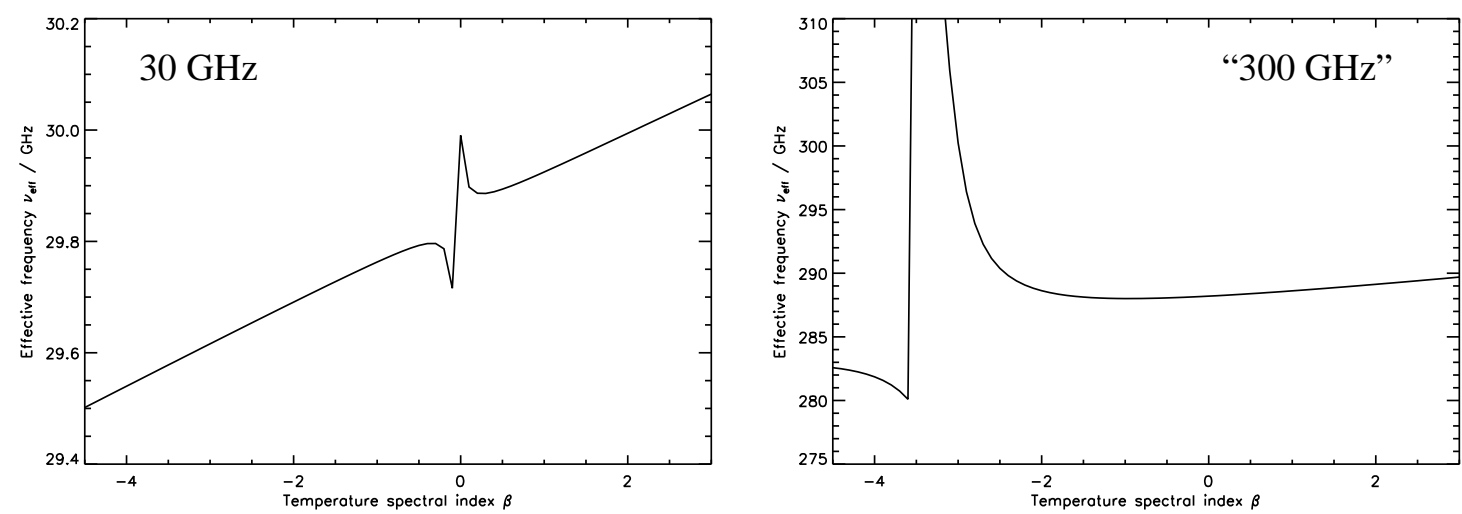

Figure 2: $v_{\text {eff }}$ vs. $\beta$ for our example bandpasses. Anomalous structure occurs when $\beta$ is close to that of the CMB fluctuations (note the steep $T_{A}$ spectrum of the CMB at $300 \mathrm{GHz}$ ): since the calibration is frequencyindependent for the $\mathrm{CMB}$, the effective frequency for similar spectra depends strongly on the detailed shape of the bandpass (and is multi-valued; we select the solution closest to the nominal frequency); however the residual gain error is then insensitive to $v_{\text {eff }}$.
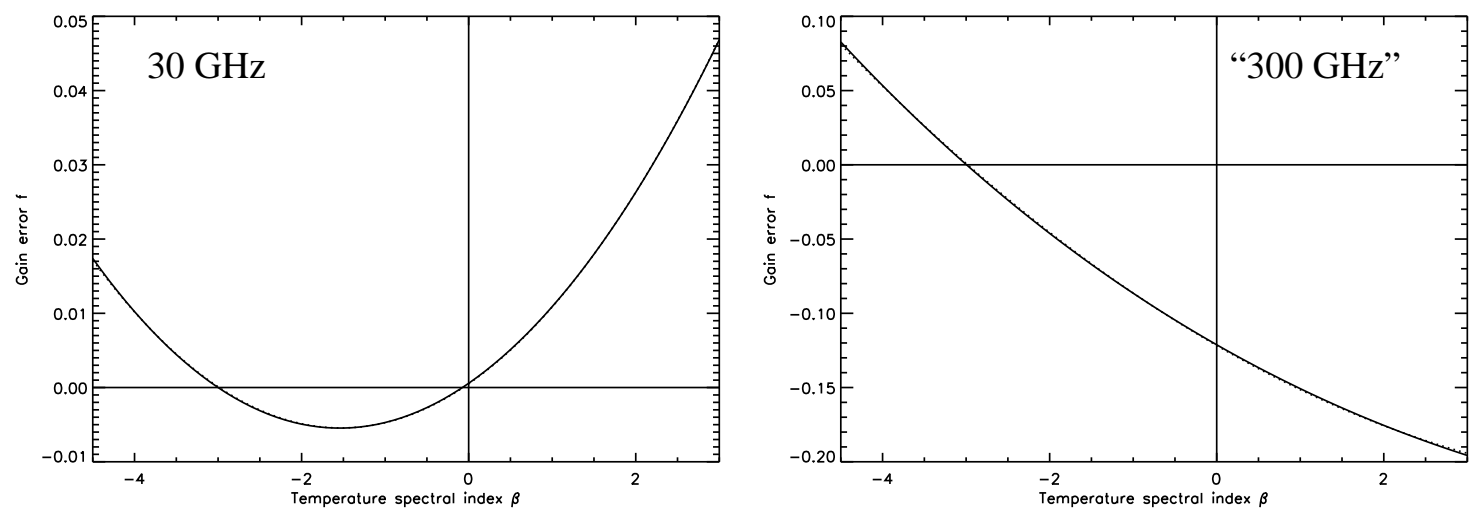

Figure 3: Residual errors $f(\beta)$ for our example bandpasses. (a) Left: For the ' $30 \mathrm{GHz}$ ' bandpass, $v_{0}$ is chosen to equal $v_{\mathrm{eff}}(\beta=-3)$, in order to give minimum residual errors for synchrotron emission. (b) Right: For the "300 GHz" bandpass we set $v_{0}$ to the target value of $300 \mathrm{GHz}$, ignoring the fact that $v_{\text {eff }}=285$ $290 \mathrm{GHz}$ for reasonable $\beta$. The offset from $v_{\text {eff }}$ gives a large linear gradient in $f(\beta)$. Solid lines are exact calculations from numerical integration across the measured bandpass; close inspection will also reveal dotted lines, which are parabolic fits and almost exactly overlay the accurate curves.

effective bandwidth. The apparent $\beta_{\mathrm{CMB}}$ is close to the actual gradient of the CMB spectrum at $30 \mathrm{GHz}$, but less so at high frequencies, due to spectral curvature within the band.

\section{Multiple Detectors: $T$ vs $P$}

In maps of $T$ made by averaging the results from several detectors, if we assign a nominal frequency equal to the average $v_{\text {eff }}$, we have $\langle\varepsilon\rangle=0$ and so the residual bandpass errors should be proportional to $b^{2} / 24$ and have the parabolic form of Fig. 3a. In contrast, in polarization maps, derived from differences between detector outputs (native Stokes $Q=[T(a)-T(b)] / 2$ ), the $b^{2}$ term 

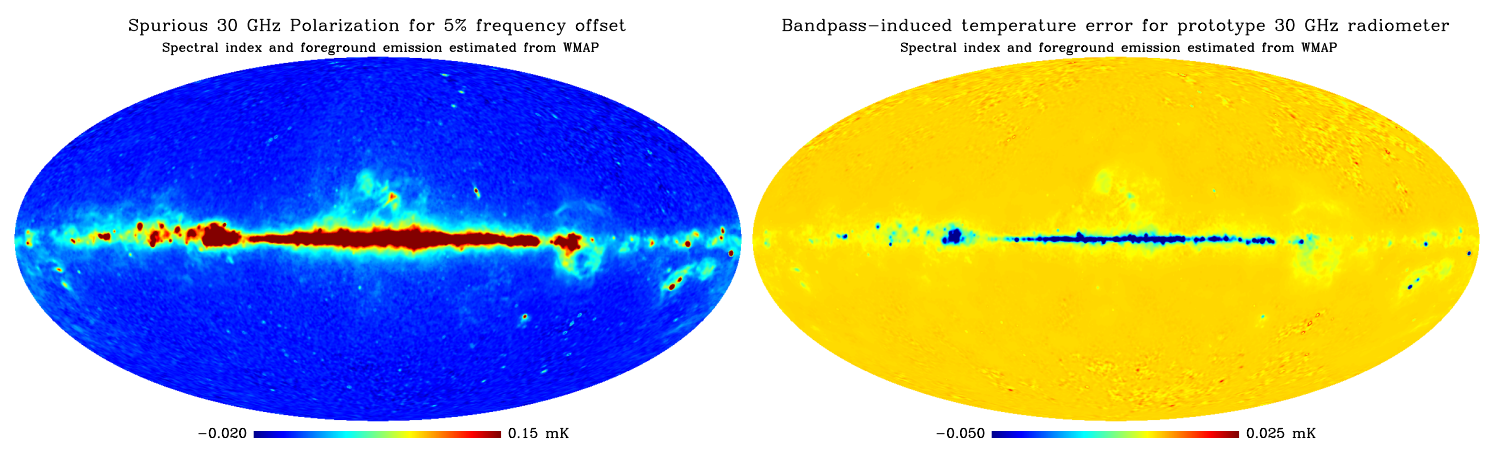

Figure 4: (a) Left: Polarization errors at $30 \mathrm{GHz}$ assuming a $5 \%$ offset between $v_{\text {eff }}$ for the ' $a$ ' and 'b' polarization radiometers. (b) Right: Temperature error expected at $30 \mathrm{GHz}$ based on WMAP data assuming the bandpass in Fig. 1a and $v_{0}$ as in Fig. 3a.

will approximately cancel, so the error term will be proportional to $\Delta \varepsilon / 2$ and be quasi-linear as in Fig. 3b. For the LFI we expect $\Delta \varepsilon \sim 3 \%$, cf. $b^{2} / 24 \sim 0.2 \%$, so the spurious polarization is by far the larger artefact, even before accounting for the much weaker signal in $P$. For the HFI, since the same filter defines the bandpass for both polarizations, we expect $f(a)-f(b)$ to be almost zero, except for the small frequency dependence of the beam and bolometers, giving very small bandpass errors in $P$.

\subsection{Estimated Error Amplitudes}

We estimated the foreground spectral index distribution from the WMAP 3-year data, and calculated the bandpass-induced errors in T and P for the LFI at $30 \mathrm{GHz}$, assuming $b=0.25$ as for the bandpass in Fig 1a, and $\Delta \varepsilon=5 \%$. These errors have rms, outside the WMAP Kp2 mask, of $0.7 \mu \mathrm{K}$ in temperature and $6.3 \mu \mathrm{K}$ in native $Q$.

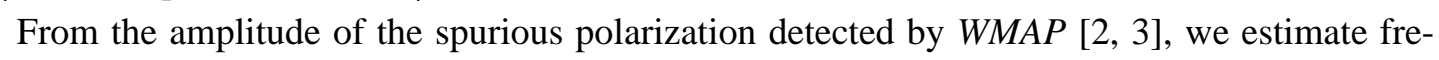
quency offsets of $\sim 1 \%$ between their two polarization channels. Improved estimates of the WMAP polarization could be obtained by using our formalism to find one frequency offset for each differencing assembly, instead of deriving an independent spurious polarization signal at each of 3072 pixels for each assembly. Our approach is required for Planck, since each pixel is only observed with two scan angles (quite similar ones for most pixels), so we cannot use the WMAP technique of identifying the spurious polarization as a native- $Q$ signal independent of scan angle.

Acknowledgements We thank D. Kettle for providing the bandpass data. K.F. thanks the EU for a Marie Curie Training Fellowship.

\section{References}

[1] Jarosik, N. et al. 2003. ApJS, 145, 413

[2] Jarosik, N. et al. 2006. ApJ, submitted, astro-ph/0603452

[3] Page, L. et al. 2006. ApJ, submitted. astro-ph/0603450 